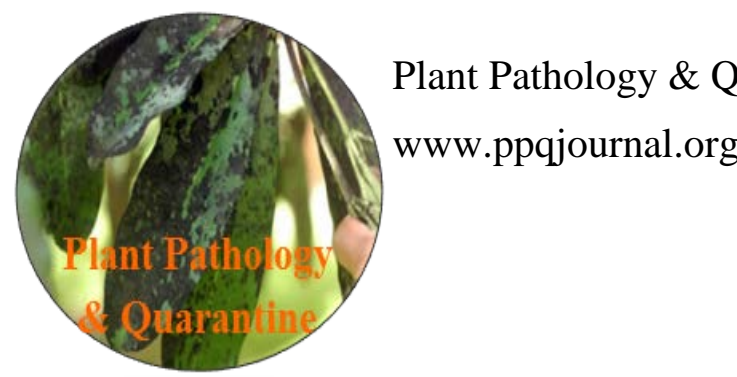

ISSN 2229-2217

\title{
An annotated list of genus Pythium from India
}

\author{
Dubey MK ${ }^{1,2 *}$, Yadav $\mathbf{M}^{1}$ and Upadhyay RS $^{1}$
}

\begin{abstract}
${ }^{1}$ Laboratory of Mycopathology and Microbial Technology, Centre of Advanced Study in Botany, Institute of Science, Banaras Hindu University, Varanasi - 221005, Uttar Pradesh, India

${ }^{2}$ Department of Life Sciences, School of Basic \& Applied Sciences, Galgotias University, Greater Noida- 203201, Uttar Pradesh, India
\end{abstract}

Dubey MK, Yadav M, Upadhyay RS 2020 - An annotated list of genus Pythium from India. Plant Pathology \& Quarantine 10(1), 120-132, Doi 10.5943/PPQ/10/1/14

\begin{abstract}
Up-to-date information is presented based on an intensive search of literature records on the identity, occurrence, nomenclature, substratum, host ranges, geographical distribution and literature references of the genus Pythium from India. All Pythium species published until 2020 are included in this list. The survey result of all forms of analyses revealed that India has 55 species of Pythium belonging to the phylum Oomycota indicating the presence of rich mycoflora. Distribution of these Pythium species reported so far from freshwater and terrestrial habitats of various Indian states are listed alphabetically. The most frequently collected species are Pythium aphanidermatum, P. spinosum, and $P$. ultimum. The majority of these species were found as a parasite on a wide range of plants in both freshwater and terrestrial environment. Overall, this systematic checklist provides the total count of Pythium species, currently known to occur in India and it is also a valued addition for comparing Pythium diversity in India as well as the world. Besides, it represents the first comprehensive overview of Pythium since 1996 from India. The knowledge generated by this working checklist comprising accepted taxa in Pythium from India is hoped to be beneficial in the progress of the systematics, diversity, ecology, plant protection, aquaculture, ichthyopathology, quarantine and many other diverse arrays of applied scientific disciplines in the country.
\end{abstract}

Key words - Disease - distribution - ecology - parasitic - soil-borne pathogen - saprobes systematic taxonomy

\section{Introduction}

The oomycete genus Pythium (Pythiaceae, Pythiales) is a large heterogeneous group currently placed under the kingdom Chromista or Heterokonta (also called Straminipila) in the supergroup Chromalveolates (Kirk et al. 2008, Beakes et al. 2014, Dubey et al. 2020a). This large genus of fungal-like organisms is characterized by profusely branched, well developed, filamentous (threadlike), coenocytic (non-septate) mycelium composed principally of cellulosic cell walls with filamentous to globose sporangia/zoosporangia like hyphal swellings containing asexually formed heterokont zoospores and sexually produced organs such as antheridia, oogonia and oospores (van der Plaats-Niterink 1981, Beakes et al. 2014). Currently, this cosmopolitan genus includes more than over 300 recorded species out of which 130 have been well-recognized (Dick 1990) with a wide range of life histories that includes many saprophytes, plant or animal pathogens and mycoparasites 
in various types of soil and aquatic environments (Lévesque \& de Cock 2004, Webster \& Weber 2007, Mufunda et al. 2017). The Pythium comprises endophytes, saprobes and plant pathogens. However, most of the species in this largest oomycete genus often serve as obligate saprotrophs on animal and plant debris; significantly helping in maintaining the natural nutrient cycling and the energy budget of the freshwater and terrestrial ecosystem around the world (Dick 2001, Dubey et al. 2020a). However, under certain favorable conditions, Pythium spp. can parasitize a wide range of hosts including algae, crustacean, fish, insects or mosquito larvae, humans beings, even other fungi or Pythium species as mycoparasites and plants (van der Plaats-Niterink 1981, Kawamura et al. 2005, Weiland et al. 2012, Ho 2013). The species belonging to Pythium are the causal agents of rot and damping-off diseases of numerous wild as well as cultivated plants (Martin \& Loper 1999, Villa et al. 2006, Broders et al. 2007). The Pythium, being obligate plant parasites, cause a variety of plant diseases or can damage to plant products, in most cases collectively accounts for multibillion-dollar losses due to the death of a large area of the world economically important cash crops (van West et al. 2003). Thus the knowledge of this most economically important group oomycete in a particular area is important concerning the biology, systematics and taxonomy as well as for phytopathological remediation purposes to prevent such diseases or reduce the losses they cause.

To our knowledge, since the earliest record by Butler (1907), many Pythium specimens have been collected from India. The vast biodiversity and climatic conditions of India contribute to the vast diversity, distribution and host range of this group. However still, a large number of the representatives of this genus have not been reported and extensively studied from India. Further, the information pertaining to this genus and their host plant association is scattered and restricted to some regional studies in various plant pathological reports and some general fungal lists. Some publications provided lists of the Pythium from India (Rao 1963, Misra \& Hall 1996), but these were incomplete, outdated and largely based on morphological data. Information regarding the occurrence and distribution of Pythium from India is difficult to obtain, primarily because literature is scattered and some of it is unavailable. Over the years with the advent of molecular biology, Pythium has undergone several taxonomic revisions, updates and a significant number of new records have been reported from India. The need for an up-to-date list is therefore evident. To remedy this, in the present contribution a comprehensive review of the species, host range and geographical distribution of Pythium in India is provided so that it will be more widely available to Indian phytopathologists and researchers interested in this ecological group. Moreover, data of earlier researchers are listed, to make this new checklist as complete as possible to serve as a baseline for future mycological studies.

\section{Materials \& Methods}

All the previously published information relevant to the genus Pythium in India were surveyed and compiled (latest accessed 10/04/2020). The checklist was primarily based on an exhaustive bibliographic survey of the literature published in various national and international journals, online available digitized records of specimens, monographs, reports, books, book chapters and even magazines that included records of Pythium spp. from India. The different collections were compiled by disposing of each single species and subspecies/variety including the name of the species in alphabetical order and the authors' epithets, host range/substrate, habitat or geographical distribution, and related recent references. Besides, from a phytopathological point of view, a family-wise list of the susceptible host plant for Pythium mediated diseases was prepared to assess their host range and variation in India. All species names were cross-checked for its validity and some preceding names as recorded in the cited publications have been substituted with their currently accepted scientific name according to the fungal nomenclature database MycoBank and Species Fungorum (http://www.mycobank.org, www.speciesfungorum.org, www.indexfungorum.org) website.

\section{Results}

A total of 55 species and 4 varieties of the genus Pythium are listed and arranged alphabetically in chronological order with their respective substrate/host range, places of collection, and related references from India (Table 1). The most frequently collected Pythium species are P. debaryanum, 
$P$. aquatile, $P$. aphanidermatum, $P$. deliense, $P$. dissotocum, $P$. graminicola, $P$. intermedium, $P$. middletonii, $P$. myriotylum, $P$. spinosum, $P$. ultimum, and $P$. vexans. While the distribution of $P$. anguillulae-aceti, P. apleroticum, P. cucurbitacearum, P. drechsleri, P. echinogynum, P. elongatum, $P$. helicoides, $P$. hypogynum, $P$. indigoferae, $P$. kashmirense, P. lobatum, P. marsipium, $P$. monospermum, $P$. multisporum, $P$. parasiticum, $P$. periilum, $P$. periplocum, $P$. polytylum, $P$. pulchrum, $P$. rhizo-oryzae, $P$. rhizosaccharum, $P$. torulosum, $P$. stipitatum and $P$. campanulatum were found to be rare. Besides, over 46 Pythium taxa were recorded over the plants either as a parasite or saprophyte. The plants belonging to the family Malvaceae, Zingiberaceae, Solanaceae, Gramineae, Zygnemataceae, Cucurbitaceae, Papaveraceae, Araceae, Fabaceae, Apiaceae, Brassicaceae, Piperaceae, Anabantidae, Rosaceae, Geraniaceae and Cyprinidae are mostly prone to Pythium infection. After compilation of the literature records, it was noticed that most of the taxa were reported from South India followed by North India, East India and West India, respectively. In between them, most reports are available from Coimbatore (Tamil Nadu), Nainital (Uttarakhand), Varanasi, Gorakhpur (Uttar Pradesh) and Hyderabad (Telangana), respectively. Neither of the taxa has been ever recorded from Indian states such as Punjab, Goa, Andhra Pradesh, Orissa, Chhattisgarh, Jharkhand, Arunachal Pradesh, Manipur, Mizoram, Nagaland, and Tripura. Even though of the above results, about one-third of all Pythium species are more or less uniformly distributed throughout India. These ubiquitous species include $P$. graminicola, $P$. insidiosum, $P$. aphanidermatum, $P$. dissotocum, $P$. myriotylum, $P$. deliense, $P$. ultimum, $P$. catenulatum, $P$. debaryanum, $P$. dissotocum, $P$. intermedium, $P$. middletonii, $P$. spinosum and $P$. vexans. The Indian checklist contains several species that have importance to humans, such as important pathogens of crops (e.g., $P$. aphanidermatum, P. acanthophoron, P. aquatile, P. catenulatum, P. debaryanum, P. deliense, $P$. dissotocum, $P$. echinogynum, $P$. echinulatum and $P$. graminicola, etc), algae ( $P$. carolinianum, $P$. catenulatum and P. cucurbitacearum), fishes (P. afertile and P. undulatum), Mosquito larva and human (e.g., $P$. insidiosum). Most of the India Pythium species were recorded from soil and water habitats whereas neither spp. was reported from the marine environment. P. acanthicum, $P$. apleroticum, $P$. drechsleri, $P$. elongatum, $P$. drechsleri, $P$. elongatum, $P$. kashmirense, $P$. lobatum, $P$. mamillatum, $P$. multisporum, $P$. parasiticum, $P$. periilum, $P$. pulchrum, $P$. rhizo-oryzae, $P$. rhizosaccharum were reported to be saprophytic in India. In contrast, $P$. acanthophoron, $P$. aphanidermatum, $P$. aquatile, $P$. carolinianum, $P$. catenulatum, $P$. debaryanum, $P$. deliense, $P$. dissotocum, $P$. echinulatum, $P$. helicoides, $P$. hydnosporum, $P$. indigoferae, $P$. irregular, $P$. middletonii, $P$. periplocum, $P$. spinosum, $P$. torulosum, $P$. vexansi, $P$. diclinum were parasitic as well as saprophytic in their mode of nutrition. In this sense, $P$. afertile, $P$. anguillulae-aceti, $P$. cucurbitacearum, $P$. echinogynum, $P$. graminicola, $P$. hypogynum, $P$. inflatum, $P$. insidiosum, $P$. intermedium, $P$. iwayamai, $P$. marsipium, $P$. monospermum, $P$. myriotylum, $P$. oedochilum, $P$. oligandrum, $P$. paroecandrum, $P$. polytylum, $P$. rostratum, $P$. splendens, $P$. ultimum, $P$. undulatum were parasitic in their mode of nutrition. The majority of the listed Pythium taxa were identified sorely based on the morphological features such as vegetative organs like the formation of hyphal swellings; asexual structures such as size and shape of zoosporangium/ heterokont zoospores, the formation of papilla/discharged tubes and patterns of their discharge; and reproductive organs including structure, production and mode of attachment of the antheridium, oogonium and oospores. However, in recent years, several Pythium spp. such as $P$. insidiosum, $P$. rhizosaccharum, $P$. aphanidermatum, $P$. dissotocum, $P$. myriotylum, $P$. deliense, $P$. rhizo-oryzae, $P$. graminicola, $P$. catenulatum, $P$. stipitatum, $P$. campanulatum and $P$. helicoides were subjected to morpho-molecular evaluation and identified largely based on molecular data.

\section{Discussion}

The current checklist is the first in a series of lists on the traditional zoosporic fungi recorded for India. The present publication aims to combine all earlier biodiversity explorations and their information pertaining to the genus Pythium in one list. The Pythium is considered one of the least explored ecological niches for oomycete remaining today in India. The research in this area has been primarily hampered by a confusing taxonomy largely dependent on nineteenth-century concepts and 
exceedingly inadequate literature. However, these water mold being an important biodiversity component have the potential of impacting global food security and the human economy. Some species of this cosmopolitan oomycete listed herewith are considered to be an important pathogen of cash crops (e.g., P. aphanidermatum, P. myriotylum, P. arrhenomanes, P. dissotocum, $P$. elongatum, and $P$. spinosum) and have been reported to cause seedling damping-off and root rot (Khulbe 2001). Owing to their economic importance, the present insight is needed to prepare an outlook for the future. In this sense, past works (1907-2020) on Pythium spp. reported from India were studied to provide a compile data on an annotated alphabetical checklist of the genus herewith.

Table 1 Pythium species recorded from India. Abbreviations: S: soil, W: water, PA: parasite, SA: saprotroph, MP: Madhya Pradesh, UP: Uttar Pradesh, KA: Karnataka, TN: Tamil Naidu, RA: Rajasthan, UK: Uttarakhand, AP: Andhra Pradesh, WB: West Bengal, GU: Gujrat, KE: Kerala, MS: Maharashtra, JK: Jammu and Kashmir, HP: Himachal Pradesh and ME: Meghalaya.

\begin{tabular}{|c|c|c|c|c|c|c|}
\hline $\begin{array}{l}\text { S. } \\
\text { No. }\end{array}$ & Pythium species & $\begin{array}{l}\text { Type of } \\
\text { sample }\end{array}$ & Substrates & Nutrition & Collection data & Reference \\
\hline 1 & $\begin{array}{l}\text { P. acanthicum } \\
\text { Drechsler }\end{array}$ & S & Agricultural soil & SA & $\begin{array}{l}\text { Hyderabad, Vakarabad } \\
\text { (Telangana); Gwalior } \\
\text { (MP); Banglore (KA) }\end{array}$ & $\begin{array}{l}\text { Joshi \& } \\
\text { Chauhan } 1982\end{array}$ \\
\hline 2 & $\begin{array}{l}\text { P. acanthophoron } \\
\text { Sideris }\end{array}$ & $\mathrm{S}$ & $\begin{array}{l}\text { Gossypium seed } \\
\text { (Malvaceae) and } \\
\text { Zingiber officinale } \\
\text { (Zingiberaceae) } \\
\text { rhizome }\end{array}$ & PA, SA & $\begin{array}{l}\text { Coimbatore (TN); } \\
\text { Udaipur (RA) }\end{array}$ & $\begin{array}{l}\text { Lodha \& } \\
\text { Webster } 1990\end{array}$ \\
\hline 3 & $\begin{array}{l}\text { P. afertile Kanouse } \\
\text { and Humphrey }\end{array}$ & W, S & $\begin{array}{l}\text { Fish eggs, infected } \\
\text { roots }\end{array}$ & PA & $\begin{array}{l}\text { Nainital (UK); } \\
\text { Varanasi (UP) }\end{array}$ & $\begin{array}{l}\text { Kiran et al. } \\
1982, \text { Khulbe } \\
1977, \\
\text { Sati } 1981\end{array}$ \\
\hline 4 & $\begin{array}{l}\text { P. anguillulae-aceti } \\
\text { Sadebeck }\end{array}$ & $\mathrm{S}$ & $\begin{array}{l}\text { Solanum } \\
\text { melongena } \\
\text { (Solanaceae) }\end{array}$ & PA & $\begin{array}{l}\text { Bomori, Haldwani } \\
\text { (UK) }\end{array}$ & Bhatt 2000 \\
\hline 5 & $\begin{array}{l}\text { P. aphanidermatum } \\
\text { (Edson) Fitzp }\end{array}$ & S & $\begin{array}{l}\text { Reported on many } \\
\text { monocotyledonous } \\
\text { and dicotyledonous } \\
\text { plants }\end{array}$ & $\mathrm{PA}, \mathrm{SA}$ & $\begin{array}{l}\text { Ubiquitous in nature } \\
\text { throughout India }\end{array}$ & $\begin{array}{l}\text { Muthukumar } \\
\text { 2010, Ashwathi } \\
\text { et al. } 2017\end{array}$ \\
\hline 6 & $\begin{array}{l}\text { P. apleroticum } \\
\text { Tokunaga }\end{array}$ & $\mathrm{W}$ & Plant debris & SA & Varanasi (UP) & $\begin{array}{l}\text { Sarkar et al. } \\
1981\end{array}$ \\
\hline 7 & P. aquatile Hohnk & $\mathrm{S}, \mathrm{W}$ & $\begin{array}{l}\text { Lycopersicon } \\
\text { esculentum } \\
\text { (Solanaceae) }\end{array}$ & SA, PA & $\begin{array}{l}\text { Gorakhpur (UP); } \\
\text { Hissar (Haryana); } \\
\text { Hyderabad (AP) }\end{array}$ & $\begin{array}{l}\text { Prabhuji \& } \\
\text { Srivastava 1978, } \\
\text { Manoharachary } \\
\text { \& Rao } 1978\end{array}$ \\
\hline 8 & $\begin{array}{l}\text { P. campanulatum } \\
\text { Mathew, Singh, and } \\
\text { Paul }\end{array}$ & $\mathrm{S}$ & $\begin{array}{l}\text { Rhizosphere of Zea } \\
\text { mays (Gramineae) }\end{array}$ & SA & Gorakhpur (UP) & $\begin{array}{l}\text { Mathew et al. } \\
2003\end{array}$ \\
\hline 9 & $\begin{array}{l}\text { P. carolinianum } \\
\text { Matthews }\end{array}$ & $\mathrm{W}, \mathrm{S}$ & $\begin{array}{l}\text { Spirogyra species } \\
\text { (Zygnemataceae) } \\
\text { and Vegetable } \\
\text { debris }\end{array}$ & PA, SA & $\begin{array}{l}\text { Coimbatore (TN); } \\
\text { Hyderabad } \\
\text { (Telangana); Prayagraj, } \\
\text { Chandauli (UP) }\end{array}$ & $\begin{array}{l}\text { Balakrishnan } \\
\text { 1948, } \\
\text { Rajagopalan \& } \\
\text { Ramakrishnan } \\
\text { 1964, 1971, } \\
\text { Dubey } 2018\end{array}$ \\
\hline 10 & $\begin{array}{l}\text { P. catenulatum } \\
\text { Matthews }\end{array}$ & $\mathrm{W}, \mathrm{S}$ & $\begin{array}{l}\text { Spirogyra species } \\
\text { (Zygnemataceae) } \\
\text { and Saccharum } \\
\text { officinarum } \\
\text { (Poaceae) }\end{array}$ & $\mathrm{PA}, \mathrm{SA}$ & $\begin{array}{l}\text { Coimbatore (TN); } \\
\text { Prayagraj, Chandauli } \\
\text { (UP) }\end{array}$ & $\begin{array}{l}\text { Balakrishnan } \\
1948, \\
\text { Srinivasan } \\
1956, \\
\text { Chona 1958, } \\
\text { Dubey } 2018\end{array}$ \\
\hline 11 & $\begin{array}{l}\text { P. cucurbitacearum } \\
\text { Takimoto }\end{array}$ & $\mathrm{S}$ & $\begin{array}{l}\text { Trichosanthes } \\
\text { dioica } \\
\text { (Cucurbitaceae) }\end{array}$ & PA & Nadia (WB) & Chaudhuri 1975 \\
\hline
\end{tabular}


Table 1 Continued.

\begin{tabular}{|c|c|c|c|c|c|c|}
\hline $\begin{array}{l}\text { S. } \\
\text { No. }\end{array}$ & Pythium species & $\begin{array}{l}\text { Type of } \\
\text { sample }\end{array}$ & Substrates & Nutrition & Collection data & Reference \\
\hline 12 & $\begin{array}{l}\text { P. debaryanum } \\
\text { Hesse }\end{array}$ & $\mathrm{S}$ & $\begin{array}{l}\text { Reported on many } \\
\text { monocotyledonous } \\
\text { and dicotyledonous } \\
\text { plants }\end{array}$ & PA, SA & $\begin{array}{l}\text { Ubiquitous in nature } \\
\text { throughout India }\end{array}$ & $\begin{array}{l}\text { Butler 1907, } \\
\text { 1913, Srivastava } \\
\text { \& Rao 1964, } \\
\text { Kapoor } 2008\end{array}$ \\
\hline 13 & P. deliense Meurs & $S$ & $\begin{array}{l}\text { Reported on many } \\
\text { dicotylednous } \\
\text { plants }\end{array}$ & PA, SA & $\begin{array}{l}\text { Ubiquitous in nature } \\
\text { throughout India }\end{array}$ & $\begin{array}{l}\text { Haware \& Joshi } \\
\text { 1974, } \\
\text { Jooju 2005, } \\
\text { Muthukumar } \\
2010\end{array}$ \\
\hline 14 & $\begin{array}{l}\text { P. diclinum } \\
\text { Tokunaga }\end{array}$ & $\mathrm{S}$ & $\begin{array}{l}\text { Reported on many } \\
\text { monocot and dicot } \\
\text { plants }\end{array}$ & SA, PA & $\begin{array}{l}\text { Surat (GU); Dehradun } \\
\text { (UK); Kolkata (WB) }\end{array}$ & Butler 1907 \\
\hline 15 & $\begin{array}{l}\text { P. dissotocum } \\
\text { Drechsler }\end{array}$ & S, W & $\begin{array}{l}\text { Papaver } \\
\text { somniferum } \\
\text { (Papaveraceae) }\end{array}$ & SA, PA & $\begin{array}{l}\text { Barabanki, Lucknow } \\
\text { (UP); New Delhi; } \\
\text { Bangalore (KA); } \\
\text { Kasargod (KE); } \\
\text { Coimbatore (TN) }\end{array}$ & $\begin{array}{l}\text { Chowdhry \& } \\
\text { Agarwal 1980, } \\
\text { 1981, Alam et } \\
\text { al. 1996, Bajpai } \\
\text { et al. } 1999\end{array}$ \\
\hline 16 & $\begin{array}{l}\text { P. drechsleri } \\
\text { Rajagopalan and } \\
\text { Ramakrishnan }\end{array}$ & $S$ & Agricultural soil & SA & Coimbatore (TN) & $\begin{array}{l}\text { Rajagopalan \& } \\
\text { Ramakrishnan } \\
1971\end{array}$ \\
\hline 17 & $\begin{array}{l}\text { P. echinogynum } \\
\text { Balghouthi, } \\
\text { Jonathan, Gognies, } \\
\text { Mliki and Belarbi }\end{array}$ & $S$ & $\begin{array}{l}\text { Turf grassroots } \\
\text { (Poaceae) }\end{array}$ & PA & Nagpur (MS) & $\begin{array}{l}\text { Balghouthi et al. } \\
2013\end{array}$ \\
\hline 18 & $\begin{array}{l}\text { P. echinulatum } \\
\text { Matthews }\end{array}$ & S & $\begin{array}{l}\text { Triticum aestivum } \\
\text { (Poaceae) }\end{array}$ & SA, PA & $\begin{array}{l}\text { Gorakhpur (UP); } \\
\text { Nainital (UK); } \\
\text { Vikarabad, Medak and } \\
\text { Hyderabad (Telangana) }\end{array}$ & $\begin{array}{l}\text { Rama Rao 1970, } \\
\text { Verma 1987a }\end{array}$ \\
\hline 19 & $\begin{array}{l}\text { P. elongatum } \\
\text { Mathews }\end{array}$ & W, S & Moist soil & SA & $\begin{array}{l}\text { Hyderabad } \\
\text { (Telangana); Nainital } \\
\text { (UK) }\end{array}$ & Khulbe 1983 \\
\hline 20 & $\begin{array}{l}\text { P. graminicola } \\
\text { Subramaniam }\end{array}$ & S & $\begin{array}{l}\text { Reported on many } \\
\text { monocotyledonous } \\
\text { and dicotyledonous } \\
\text { plants }\end{array}$ & PA & $\begin{array}{l}\text { Pusa (Bihar); New } \\
\text { Delhi; Coimbatore, } \\
\text { Chidambaram (TN); } \\
\text { Jabalpur (MP); } \\
\text { Chandauli (UP) }\end{array}$ & $\begin{array}{l}\text { Subramaniam } \\
1928, \\
\text { Ramakrishnan } \\
\text { \& Soumini } \\
\text { 1955, } \\
\text { Muthukumar } \\
\text { 2010, } \\
\text { Dubey et al. } \\
\text { 2020a }\end{array}$ \\
\hline 21 & $\begin{array}{l}\text { P. helicoides } \\
\text { Drechsler }\end{array}$ & $\mathrm{S}$ & $\begin{array}{l}\text { Amorphophallus } \\
\text { paeoniifolius } \\
\text { (Araceae) }\end{array}$ & $\mathrm{SA}, \mathrm{PA}$ & $\begin{array}{l}\text { Coimbatore (TN), } \\
\text { Nadia (WB) }\end{array}$ & $\begin{array}{l}\text { Guha Roy \& } \\
\text { Hong } 2008\end{array}$ \\
\hline 22 & $\begin{array}{l}\text { P. hydnosporum } \\
\text { (Montagne) } \\
\text { Schroter }\end{array}$ & $\mathrm{S}, \mathrm{W}$ & $\begin{array}{l}\text { Solanum tuberosum } \\
\text { (Solanaceae) }\end{array}$ & SA, PA & $\begin{array}{l}\text { Kolkata (WB); Khasi } \\
\text { Hills (Assam); } \\
\text { Coonoor (TN) }\end{array}$ & $\begin{array}{l}\text { Sydow \& Butler } \\
\text { 1907, } \\
\text { Chowdhry \& } \\
\text { Agarwal } 1980\end{array}$ \\
\hline 23 & $\begin{array}{l}P . \text { hypogynum } \\
\text { Middleton }\end{array}$ & S, W & $\begin{array}{l}\text { Triticum aestivum } \\
\text { (Poaceae) }\end{array}$ & PA & $\begin{array}{l}\text { Udham Singh Nagar } \\
\text { (UK) }\end{array}$ & Verma 1984 \\
\hline 24 & $\begin{array}{l}\text { P. indigoferae } \\
\text { Butler }\end{array}$ & S & $\begin{array}{l}\text { Epiphyte on the } \\
\text { leaves of Indigofera } \\
\text { arrecta (Fabaceae) } \\
\text { and Cucumis } \\
\text { sativus } \\
\text { (Cucurbitaceae) }\end{array}$ & SA, PA & Kolkata (WB) & Butler 1907 \\
\hline
\end{tabular}


Table 1 Continued.

\begin{tabular}{|c|c|c|c|c|c|c|}
\hline $\begin{array}{l}\text { S. } \\
\text { No. }\end{array}$ & Pythium species & $\begin{array}{l}\text { Type of } \\
\text { sample }\end{array}$ & Substrates & Nutrition & Collection data & Reference \\
\hline 25 & $\begin{array}{l}P . \text { inflatum } \\
\text { Matthews }\end{array}$ & $\mathrm{S}$ & $\begin{array}{l}\text { Oryza spp. } \\
\text { (Poaceae) and } \\
\text { Lycopersicon } \\
\text { esculentum } \\
\text { (Solanaceae) }\end{array}$ & PA & $\begin{array}{l}\text { Hyderabad } \\
\text { (Telangana); Nainital } \\
\text { (UP) }\end{array}$ & $\begin{array}{l}\text { Verma \& } \\
\text { Khulbe 1986, } \\
\text { Verma 1987b }\end{array}$ \\
\hline 26 & $\begin{array}{l}\text { P. insidiosum De } \\
\text { Cock }\end{array}$ & $\mathrm{W}$ & $\begin{array}{l}\text { Mosquito larva, } \\
\text { human }\end{array}$ & PA & $\begin{array}{l}\text { Pondicherry; } \\
\text { Hyderabad } \\
\text { (Telangana); Madurai } \\
\text { (TN) }\end{array}$ & $\begin{array}{l}\text { Schurko et al. } \\
\text { 2004, Kalra et } \\
\text { al. 2018, Hasika } \\
\text { et al. } 2019\end{array}$ \\
\hline 27 & $\begin{array}{l}\text { P. intermedium De } \\
\text { Bary }\end{array}$ & $\mathrm{S}$ & $\begin{array}{l}\text { Reported on many } \\
\text { monocot and dicot } \\
\text { plants }\end{array}$ & PA & $\begin{array}{l}\text { Nagpur (MS); } \\
\text { Bangalore (KA); } \\
\text { Peechi (KE) }\end{array}$ & $\begin{array}{l}\text { Ali \& Nair } \\
\text { 1989, Rao } 1963\end{array}$ \\
\hline 28 & $\begin{array}{l}\text { P. irregulare } \\
\text { Buisman }\end{array}$ & $\mathrm{S}$ & $\begin{array}{l}\text { Coriandrum } \\
\text { sativum (Apiaceae) }\end{array}$ & $\mathrm{SA}, \mathrm{PA}$ & $\begin{array}{l}\text { Lucknow, Varanasi } \\
\text { (UP); Bangalore (KA) }\end{array}$ & $\begin{array}{l}\text { Agnihotri 1969, } \\
\text { Sharma \& Basu } \\
\text { Chaudhary } 1981\end{array}$ \\
\hline 29 & P. iwayamai Ito & $\mathrm{S}$ & $\begin{array}{l}\text { Lycopersicon } \\
\text { esculentum and } \\
\text { Solanum } \\
\text { melongena } \\
\text { (Solanaceae) }\end{array}$ & PA & $\begin{array}{l}\text { Bomori, Haldwani, } \\
\text { Nainital, Rooshi (UK) }\end{array}$ & Bhatt 2000 \\
\hline 30 & P. kashmirense Paul & $S$ & Plant debris & SA & Reasi (JK) & $\begin{array}{l}\text { Paul \& Bala } \\
2008\end{array}$ \\
\hline 31 & $\begin{array}{l}\text { P. lobatum } \\
\text { Rajagopalan and } \\
\text { Ramakrishnan }\end{array}$ & S & Moist soil & SA & Coimbatore (TN) & $\begin{array}{l}\text { Rajagopalan \& } \\
\text { Ramakrishnan } \\
1971\end{array}$ \\
\hline 32 & $\begin{array}{l}\text { P. mamillatum } \\
\text { Meurs }\end{array}$ & $S$ & Moist soil & SA & $\begin{array}{l}\text { Chennai (TN); Pakhal, } \\
\text { Medak and Hyderabad } \\
\text { (Telangana) }\end{array}$ & $\begin{array}{l}\text { Ramakrishnan } \\
\text { 1955, Rao 1963, } \\
\text { Rama Rao } 1970\end{array}$ \\
\hline 33 & $\begin{array}{l}\text { P. marsupium } \\
\text { Drechsler }\end{array}$ & S & $\begin{array}{l}\text { Solanum } \\
\text { melongena } \\
\text { (Solanaceae) }\end{array}$ & PA & $\begin{array}{l}\text { Bithoria, Haldwani } \\
\text { (UK) }\end{array}$ & Bhatt 2000 \\
\hline 34 & $\begin{array}{l}\text { P. middletonii } \\
\text { Sparrow }\end{array}$ & S, W & $\begin{array}{l}\text { Reported on many } \\
\text { dicotylednous } \\
\text { plants }\end{array}$ & $\mathrm{SA}, \mathrm{PA}$ & $\begin{array}{l}\text { Ubiquitous in nature } \\
\text { throughout India }\end{array}$ & $\begin{array}{l}\text { Singh \& Pavgi } \\
\text { 1974, Khulbe \& } \\
\text { Bhargava } 1977\end{array}$ \\
\hline 35 & $\begin{array}{l}\text { P. monospermum } \\
\text { Pringsheim }\end{array}$ & W & $\begin{array}{l}\text { Zingiber officinale } \\
\text { (Zingiberaceae) and } \\
\text { Lepidium sativum } \\
\text { (Brassicaceae) }\end{array}$ & PA & $\begin{array}{l}\text { Pusa (Bihar); Jabalpur } \\
\text { (MP); Nainital (UK) }\end{array}$ & $\begin{array}{l}\text { Rao 1963, } \\
\text { Khulbe \& } \\
\text { Bhargava } 1977\end{array}$ \\
\hline 36 & $\begin{array}{l}\text { P. multisporum } \\
\text { Poitras }\end{array}$ & S & Moist soil & SA & Kushinagar (UP) & $\begin{array}{l}\text { Prabhuji \& } \\
\text { Srivastava 1978, } \\
\text { Prabhuji \& } \\
\text { Sinha } 1994\end{array}$ \\
\hline 37 & $\begin{array}{l}\text { P. myriotylum } \\
\text { Drechsler }\end{array}$ & $\mathrm{W}, \mathrm{S}$ & $\begin{array}{l}\text { Reported on many } \\
\text { monocot and dicot } \\
\text { plants }\end{array}$ & PA & $\begin{array}{l}\text { Ubiquitous in nature } \\
\text { throughout India }\end{array}$ & $\begin{array}{l}\text { Devaki et al. } \\
\text { 1991, Kumar et } \\
\text { al. 2008, Geethu } \\
\text { et al. } 2013\end{array}$ \\
\hline 38 & $\begin{array}{l}\text { P. oedochilum } \\
\text { Drechsler }\end{array}$ & S & $\begin{array}{l}\text { Brasssica oleracea } \\
\text { var. botrytis } \\
\text { (Brassicaceae) }\end{array}$ & PA & $\begin{array}{l}\text { Annamalainagar and } \\
\text { Coimbatore (TN) }\end{array}$ & $\begin{array}{l}\text { Raghunathan } \\
\text { 1968, } \\
\text { Rajagopalan \& } \\
\text { Ramakrishnan } \\
\text { 1964, 1971 }\end{array}$ \\
\hline 39 & $\begin{array}{l}\text { P. oligandrum } \\
\text { Drechsler }\end{array}$ & S & $\begin{array}{l}\text { Piper betle } \\
\text { (Piperaceae) and } \\
\text { Anabas testudineus } \\
\text { (Anabantidae) }\end{array}$ & PA & $\begin{array}{l}\text { Lucknow, Gorakhpur } \\
\text { and Varanasi (UP) }\end{array}$ & $\begin{array}{l}\text { Srivastava et al. } \\
2017 a, b\end{array}$ \\
\hline 40 & $\begin{array}{l}\text { P. parasiticum } \\
\text { Rajagopalan and } \\
\text { Ramakrishnan }\end{array}$ & S & Agricultural soil & SA & Chennai (TN) & $\begin{array}{l}\text { Rajagopalan \& } \\
\text { Ramakrishnan } \\
1971\end{array}$ \\
\hline
\end{tabular}


Table 1 Continued.

\begin{tabular}{|c|c|c|c|c|c|c|}
\hline $\begin{array}{l}\text { S. } \\
\text { No. }\end{array}$ & Pythium species & $\begin{array}{l}\text { Type of } \\
\text { sample }\end{array}$ & Substrates & Nutrition & Collection data & Reference \\
\hline 41 & $\begin{array}{l}\text { P. paroecandrum } \\
\text { Drechsler }\end{array}$ & $\mathrm{S}, \mathrm{W}$ & $\begin{array}{l}\text { Phaseolus vulgaris } \\
\text { (Fabaceae) } \\
\text { Vegetable root }\end{array}$ & PA & $\begin{array}{l}\text { Nainital and } \\
\text { Nanakmatta (UK); } \\
\text { Coimbatore (TN) }\end{array}$ & $\begin{array}{l}\text { Balakrishnan } \\
\text { 1948, } \\
\text { Verma } 1984\end{array}$ \\
\hline 42 & $\begin{array}{l}\text { P. periilum } \\
\text { Drechsler }\end{array}$ & S & $\begin{array}{l}\text { Brassica oleracea } \\
\text { var. capitata, } \\
\text { Brassica oleracea } \\
\text { var. botrytis } \\
\text { (Brassicaceae) }\end{array}$ & SA & - & $\begin{array}{l}\text { Plaats-Niterink } \\
1981\end{array}$ \\
\hline 43 & $\begin{array}{l}\text { P. periplocum } \\
\text { Drechsler }\end{array}$ & S, W & Agricultural soil & SA, PA & Coimbatore (TN) & $\begin{array}{l}\text { Balakrishnan } \\
\text { 1948, } \\
\text { Rajagopalan \& } \\
\text { Ramakrishnan } \\
\text { 1964, 1971, } \\
\text { Bisht et al. } 1997\end{array}$ \\
\hline 44 & $\begin{array}{l}\text { P. periplocum var. } \\
\text { coimbatorense } \\
\text { Balakrishnan }\end{array}$ & S & Vegetable debris & SA & Coimbatore (TN) & $\begin{array}{l}\text { Balakrishnan } \\
1948\end{array}$ \\
\hline 45 & $\begin{array}{l}\text { P. polytylum } \\
\text { Drechsler }\end{array}$ & S & Vegetable debris & PA & $\begin{array}{l}\text { Solan (HP); Nainital } \\
\text { (UK) }\end{array}$ & $\begin{array}{l}\text { Verma \& } \\
\text { Khulbe } 1985\end{array}$ \\
\hline 46 & $\begin{array}{l}\text { P. pulchrum } \\
\text { Minden }\end{array}$ & S, W & $\begin{array}{l}\text { Malus sp. } \\
\text { (Rosaceae), Crop } \\
\text { field }\end{array}$ & SA & Nainital (UK) & Mer 1982 \\
\hline 47 & $\begin{array}{l}\text { P. rhizo-oryzae } \\
\text { Paul }\end{array}$ & S & $\begin{array}{l}\text { Hibiscus esculenta } \\
\text { (Malvaceae) }\end{array}$ & SA & Gorakhpur (UP) & Bala et al. 2006 \\
\hline 48 & $\begin{array}{l}\text { P. rhizosaccharum } \\
\text { Singh, Mathew, } \\
\text { Masih and Paul }\end{array}$ & S & $\begin{array}{l}\text { The rhizosphere of } \\
\text { Oryza sativa } \\
\text { (Poaceae) }\end{array}$ & SA & Gorakhpur (UP) & $\begin{array}{l}\text { Singh et al. } \\
2003\end{array}$ \\
\hline 49 & P. rostratum Butler & S & $\begin{array}{l}\text { Saccharum } \\
\text { officinarum } \\
\text { (Poaceae) }\end{array}$ & PA & $\begin{array}{l}\text { Pusa (Bihar); Nainital } \\
\text { (UP) }\end{array}$ & $\begin{array}{l}\text { Butler 1907, } \\
\text { Verma \& } \\
\text { Khulbe } 1985\end{array}$ \\
\hline 50 & $\begin{array}{l}\text { P. spinosum } \\
\text { Sawada }\end{array}$ & $\mathrm{S}, \mathrm{W}$ & $\begin{array}{l}\text { Triticum aestivum } \\
\text { (Poaceae) }\end{array}$ & SA, PA & $\begin{array}{l}\text { Ubiquitous in nature } \\
\text { throughout India }\end{array}$ & $\begin{array}{l}\text { Rama Rao 1970, } \\
\text { Manoharachary } \\
\text { \& Reddy 1975, } \\
\text { Sati \& Tiwari } \\
1992\end{array}$ \\
\hline 51 & P. splendens Braun & S & $\begin{array}{l}\text { Reported on many } \\
\text { monocotyledonous } \\
\text { and dicotyledonous } \\
\text { plants }\end{array}$ & PA & $\begin{array}{l}\text { Lucknow (UP); } \\
\text { Hesaraghatta (KA); } \\
\text { Shillong (ME); } \\
\text { Sirmaur (HP) }\end{array}$ & $\begin{array}{l}\text { Shanmugam et } \\
\text { al. } 2010\end{array}$ \\
\hline 52 & $\begin{array}{l}\text { P. torulosum Coker } \\
\text { and Patterson }\end{array}$ & $\mathrm{W}$ & $\begin{array}{l}\text { Pelargonium } \\
\text { graveolens } \\
\text { (Geraniaceae), } \\
\text { Zingiber officinale } \\
\text { (Zingiberaceae) }\end{array}$ & SA, PA & $\begin{array}{l}\text { Varanasi (UP); Nainital } \\
\text { (UK) }\end{array}$ & $\begin{array}{l}\text { Khulbe \& } \\
\text { Verma } 1983\end{array}$ \\
\hline 53 & P. ultimum Trow & S & $\begin{array}{l}\text { Vegetable debris } \\
\text { and Triticum } \\
\text { aestivum (Poaceae) }\end{array}$ & PA & $\begin{array}{l}\text { Salon (HP); Parbhani } \\
(\mathrm{MH})\end{array}$ & $\begin{array}{l}\text { Hudge \& } \\
\text { Deshpande } \\
\text { 2014, Hudge et } \\
\text { al. 2016, Kumar } \\
\text { et al. } 2018\end{array}$ \\
\hline 54 & $\begin{array}{l}\text { P. ultimum Trow } \\
\text { var. ultimum }\end{array}$ & S & $\begin{array}{l}\text { Solanum } \\
\text { lycopersicum } \\
\text { (Solanaceae) and } \\
\text { Glycine max } \\
\text { (Fabaceae) }\end{array}$ & SA, PA & $\begin{array}{l}\text { Ubiquitous in nature } \\
\text { throughout India }\end{array}$ & $\begin{array}{l}\text { Dohroo 1987, } \\
\text { Muthukumar } \\
2010\end{array}$ \\
\hline 55 & $\begin{array}{l}\text { P. ultimum var. } \\
\text { sporangiiferum } \\
\text { Drechsler }\end{array}$ & S & $\begin{array}{l}\text { Reported on many } \\
\text { monocotyledonous } \\
\text { and dicotyledonous } \\
\text { plants }\end{array}$ & PA & $\begin{array}{l}\text { Haldwani, Ramnagar, } \\
\text { Ranikhet, Someshwar, } \\
\text { Rooshi, Nainital (UK) }\end{array}$ & Bisht et al. 1997 \\
\hline
\end{tabular}


Table 1 Continued.

\begin{tabular}{|c|c|c|c|c|c|c|}
\hline $\begin{array}{l}\text { S. } \\
\text { No. }\end{array}$ & Pythium species & $\begin{array}{l}\text { Type of } \\
\text { sample }\end{array}$ & Substrates & Nutrition & Collection data & Reference \\
\hline 56 & $\begin{array}{l}\text { P. undulatum } \\
\text { Petersen }\end{array}$ & $\mathrm{W}$ & Vegetable seedlings & PA & Nainital (UK) & $\begin{array}{l}\text { Khulbe \& } \\
\text { Bhargava 1977, } \\
\text { Sati 1991, Sati } \\
\text { \& Tiwari 1992 }\end{array}$ \\
\hline 57 & P. vexans de Bary & $\mathrm{S}$ & $\begin{array}{l}\text { Carassus auratus } \\
\text { (Cyprinidae) and } \\
\text { Brassica campestris } \\
\text { (Solanaceae), } \\
\text { Temperate fish }\end{array}$ & SA, PA & $\begin{array}{l}\text { Ubiquitous in nature } \\
\text { throughout India }\end{array}$ & $\begin{array}{l}\text { Dastur 1935, } \\
\text { Ramakrishnan } \\
\text { 1949, Wilson \& } \\
\text { Rahim } 1978\end{array}$ \\
\hline 58 & $\begin{array}{l}P . \text { vexans var. } \\
\text { minuta Mer and } \\
\text { Khulbe }\end{array}$ & S, W & $\begin{array}{l}\text { Reported on many } \\
\text { monocotyledonous } \\
\text { and dicotyledonous } \\
\text { plants }\end{array}$ & SA & Nainital (UK) & $\begin{array}{l}\text { Mer \& Khulbe } \\
1983\end{array}$ \\
\hline 59 & $\begin{array}{l}\text { Pythium stipitatum } \\
\text { Karaca and Paul }\end{array}$ & $\mathrm{S}$ & $\begin{array}{l}\text { Grass or Cyanodon } \\
\text { dactylon roots } \\
\text { (Poaceae) }\end{array}$ & SA & Nagpur (MH) & $\begin{array}{l}\text { Karaca et al. } \\
2009\end{array}$ \\
\hline
\end{tabular}

Research related to Pythium started in the early $19^{\text {th }}$ century with collections made by Butler (1907) in India. Studies about Pythium in India were intensified between the years 1950-1990. A checklist of Indian Pythium taxa was published by Misra \& Hall (1996) which included 40 species and included a survey of all known taxa and all relevant literature until that time. Since then, a large amount of new information on taxonomy, distribution, and to a lesser extent ecology, has been published. This information is contained in many papers in various journals and books, some of them not easily accessible so that a new survey is necessary. Moreover, the yearly output of publications on the subject was increasing rapidly, so that an updated version of the bibliography and a checklist seemed necessary.

Most studies on Pythium in India have been conducted primarily from South India and Kumaon Himalayan region in North India (Khulbe 2001), and a total of 46 species have been recorded from these regions of the country. Moreover, the list also contains some saprophytes and some well-proven biological control agents among them. However, little research has been undertaken phytopathogenesis mediated negative attributes on the economy and food security by this necrotrophic generalistic pathogenic Pythium flora of fields worldwide, especially in India. It is therefore pertinent to understand the Pythium diversity and associated plant diseases, which will be helpful to develop relevant action plans for the future.

Most of the Pythium were identified based on their morphological features whereas little were studied from the molecular identification point of view. Identifying the species of Pythium sorely based on morphological features has always been problematic due to various reasons, such as variations of a specific morphological feature, difficulty in isolating certain species and the lack of molecular identification data for species. Due to these reasons, identifying Pythium species based on morphological features has been a constant problem for even the most experienced mycologists (Lévesque \& de Cock 2004). Therefore, during the last decade, molecular techniques have significantly assisted in the identification of unknown Pythium species and a large number of additional new species records and new species have been added based on it. Further, based on the data present in the list, it can be concluded that still large areas in India are unexplored relative to Pythium biodiversity and studies in this field have been hampered by grossly inadequate literature and a confused taxonomy based largely on nineteenth-century concepts. It is equally likely that a large number of species have not yet been described because newly explored areas always yield new taxa. Besides, according to the recent primarily checklist of fungi of Gujarat state, India, the genus Pythium is never been recorded in Gujarat (Rajput et al. 2015). However, our list provided supporting evidence that this genus was reported from Gujrat (Butler 1907). According to our results, this oomycete parasitized a large no. of the host plant and animal species in this country like other 
members of Oomycota (Dubey et al. 2018, 2019, 2020b). However, it is expected that more collections and further taxonomic studies will substantially increase our knowledge and provide a better understanding of their biology, ecological aspects, host-specificity, origin and divergence as well as the application of this oomycete in biocontrol. Therefore, it seems a prerequisite to launch a full-scale survey of these biologically intriguing group of oomycete in other parts of India to complete at least a modicum of their checklist. After that plenty of knowledge can be accumulated that would be useful in the compilation of mycological diversity in India.

\section{Conclusion}

The current checklist facilities access to the scattered Indian literature on the topic that may not be readily available to the student of Indian mycology and the international community. Besides, the correctly identified checklist is essential, as it can assist in gathering information pertaining to the historical development in the study of Pythium and its diversity in India. The exact knowledge of this oomycete diversity is important because these water molds are the most-significant decomposers of plant and animal originated complex organic materials and normally comprise a major proportion of total microbial biomass. Nevertheless, the recent impact of global climate change and the betterknown role of mycobiota activities in the biogeochemical cycling of elements have enforced the importance of assessing the position of Indian mycobiota and its diversity. Further, the pathogenic species mentioned in the list can assist phytopathologists to confidently name disease causal agents, lead quarantine to put in place effective measures to prevent the entry of unwanted species, allow plant breeders to breed resistant varieties and biochemists to confidently put names to species producing novel chemicals. This shows clearly that the compilation of the checklist has fulfilled its purpose to encourage Indian mycologists to search for additional taxa. Besides, this checklist will help in making the evaluations of the oomycetes recorded in the country.

\section{Acknowledgments}

We thank the Head and Programme Coordinator, Department of Botany, Banaras Hindu University, Varanasi, Uttar Pradesh, India for providing library facilities.

\section{References}

Agnihotri VP. 1969 - Production and germination of appressoria in Pythium irregulare. Mycologia 61, 967-980.

Alam M, Sattar A, Chourasia HK, Janardhanan KK. 1996 - Damping-off, a new disease of opium poppy caused by Pythium dissotocum. Indian Phytopathology 49, 94-97.

Ali MM, Nair NG. 1989 - Pythium intermedium causing root rot of Oroxylum indicum in Kerala- a new Indian record. Current Science 58(13), 747.

Ashwathi S, Ushamalini C, Parthasarathy S, Nakkeeran S. 2017 - Morphological, pathogenic and molecular characterisation of Pythium aphanidermatum: A causal pathogen of coriander damping-off in India. The Pharma Innovation Journal 6(11), 44-48.

Bajpai S, Gupta MM, Kumar S. 1999 - Identification of Indian landraces of opium poppy Papaver somniferum resistant to damping-off and downy mildew fungal diseases. Journal of Phytopathology 147, 535-538.

Bala K, Gautam N, Paul B. 2006 - Pythium rhizo-oryzae sp. nov. isolated from paddy fields: taxonomy, ITS region of rDNA, and comparison with related species. Current Microbiology 52, 102-107.

Balakrishnan MS. 1948 - South Indian Phycomycetes-II. Proceedings of the National Academy of Sciences, India-Section B, 27-34.

Balghouthi A, Jonathan R, Gognies S, Mliki A et al. 2013 - A new species, Pythium echinogynum, causing severe damping-off of tomato seedlings, isolated from Tunisia, France, and India: morphology, pathology, and biological control. Annals of Microbiology 63, 253-258. 
Beakes GW, Honda D, Thines M. 2014 - Systematics of the Straminipila: Labyrinthulomycota, Hyphochytriomycota and Oomycota. In: The Mycota VIII Part A. McLaughlin, D. J. and Spatafora, J. W. (Eds.), Springer-Verlag Berlin 39-97.

Bhatt A. 2000 - Studies on Pythium species in relation to their parasitic association with Solaneous vegetables of Haldwani and Nainital (Kumaun Himalaya). Ph.D. Thesis, Kumaun University, Nainital.

Bisht GS, Joshi C, Bisht D, Khobe RD. 1997 - Distribution and pathogenicity of Pythium spp. from tomato. Indian Phytopathology 50(1), 83-97.

Broders KD, Lipps PE, Paul PA, Dorrance AE. 2007 - Characterization of Pythium spp. associated with corn and soybean seed and seedling disease in Ohio. Plant Disease 91, 727-735.

Butler EJ. 1907 - An account of the genus Pythium and some Chytridiaceae. Memoirs of the Department of Agriculture in India, Botanical Series 1(5), 1-162.

Butler EJ. 1913 - Pythium de Baryanum Hesse. Memoirs of the Department of Agriculture in India, Botanical Series 5, 262-267.

Chaudhuri S. 1975 - Fruit rot of Trichosanthes dioica L. caused by Pythium cucurbitacearum Takimoto in West Bengal. Current Science 44, 68.

Chona BL. 1958 - Some diseases of Sugarcane reported from India in recent years. Indian Phytopathology 11(1), 1-9.

Chowdhry PN, Agarwal GP. 1981 - Taxonomic studies on aquatic fungi of India. I. Pythiaceae. Indian Phytopathology 34(2), 235-236.

Chowdhry PN, Agrawal GP. 1980 - Studies on distribution of some aquatic fungi in India. Indian Phytopathology 33(1), 107-109.

Dastur JF. 1935 - Diseases of Pan (Piper betle L.) in the Central Provinces. Proc Indian Acad Sci B1: 778-815.

Devaki NS, Bhat SS, Shenoi MM, Wajid SMA. 1991 - Pythium myriotylum a new threat to tobacco in India. Indian Phytopathology 44(4), 541-542.

Dick MW. 1990 - Key to Pythium. College of Estate Management, Reading.

Dick MW. 2001 - Straminipilous Fungi. Kluwer Academic Publishers, Dordrecht.

Dohroo NP. 1987 - Pythium ultimum on Zingiber officinale. Indian Phytopathology 40, 275.

Dubey MK, Gajbhiye MH, Upadhyay RS. 2020b - Achlya bisexualis (Achlyaceae, Saprolegniales, Oomycota) - A new record for India. Nova Hedwigia 111(1-2), 101-114.

Dubey MK, James TY, Zehra A, Aamir M et al. 2019 - First record of Newbya recurva (Saprolegniaceae) from India. Nova Hedwigia 109(1-2), 81-93.

Dubey MK, Zehra A, Aamir M, Swarnmala S et al. 2020a - Isolation, identification, carbon utilization profile and control of Pythium graminicola, the causal agent of chilli damping-off. Journal of Phytopathology 168(2), 88-102.

Dubey MK, Zehra A, Meena M, Upadhyay RS. 2018. - Taxonomic note on a rare fish infecting freshwater mould Achlya ambisexualis Raper 1939 (Achlyaceae) isolated from Chandraprabha dam, Uttar Pradesh, India. Indian Journal of Fisheries 65(1), 71-78.

Dubey MK. 2018 - Isolation and characterization of zoosporic fungi from various locations of North India. Ph.D. thesis, Banaras Hindu University, Varanasi, India.

Geethu C, Resna AK, Nair RA. 2013 - Characterization of major hydrolytic enzymes secreted by Pythium myriotylum, causative agent for soft rot disease. Antonie Van Leeuwenhoek 104(5), 749-757.

Guha RS, Bhattacharyya S, Mukherjee SK, Mondal N et al. 2006 - Phytophthora melonis associated with fruit and vine rot disease of pointed gourd in India as revealed by RFLP and sequencing of ITS region. Journal of Phytopathology 154, 612-615.

Hasika R, Lalitha P, Radhakrishnan N, Rameshkumar G et al. 2019 - Pythium keratitis in South India: incidence, clinical profile, management, and treatment recommendation. Indian Journal of Ophthalmology 67(1), 42-47.

Haware MP, Joshi LK. 1974 - Studies on soft rot of ginger from Madhya Pradesh. Handbook. U.S. Department of Agriculture 262, 1-40. 
Ho HH. 2013 - The genus Pythium in mainland China. Mycosystemata 32, 20-44.

Hudge BV, Desai PP, Deshpande GD. 2016 - Screening of soybean germplasm for resistance to damping off caused by Pythium ultimum. Indian Phytopathology 69(3), 247-252.

Hudge BV, Deshpande GD. 2014 - Influence of seed exudation on pre-emergence damping off in soybean. Indian Phytopathology 67(2), 197-198.

Jooju B. 2005 - Evaluation of genetic diversity of Pythium spp. causing soft of ginger using phenotypic and molecular methods. MPhil Thesis, Bharathidasan University, Thiruchirapalli, Tamil Naidu.

Joshi IJ, Chauhan RKS. 1982 - Distribution of soil microfungi in various soil types of Chambal Ravines. Proceedings of the Indian National Science Academy 4, 525-533.

Kalra P, Bagga B, Garg P. 2018 - Pythium Insidiosum Keratitis: Histopathology and Rapid Novel Diagnostic Staining Technique. Cornea 37(3), e14.

Kapoor AS. 2008 - Biocontrol potential of Trichoderma spp. against important soilborne diseases of vegetable crops. Indian Phytopathology 61(4), 492-498.

Karaca G, Jonathan R, Paul B. 2009 - Pythium stipitatum sp. nov. isolated from soil and plant debris taken in France, Tunisia, Turkey, and India. FEMS Microbiology Letters 295, 164-169.

Kawamura Y, Yokoo K, Tojo M, Hishiike M. 2005 - Distribution of Pythium porphyrae, the causal agent of red rot disease of Porphyra spp., in the Ariake Sea, Japan Plant Disease 89, 10411047.

Khulbe RD. 1977 - Taxonomic and ecological studies of water moulds in Nainital and its suburbs. Ph.D. Thesis, Agra University, Agra.

Khulbe RD. 1985 - Studies in aquatic Phycomycetes of Nainital in relation to taxonomy, physiology, ecology and pathology. D.Sc. thesis, Kumaun University, Nainital, India.

Khulbe RD. 2001 - A Manual of Aquatic Fungi. Daya Publisher House, New Delhi.

Khulbe RD, Bhargava KS. 1977 - Distribution and seasonal periodicity of water molds in some lakes in Nainital Hills, India. Hydrobiologia 54(1), 67-72.

Khulbe RD, Bhargava KS. 1983 - Frequency of watermolds in relation to nitrate, sulphate and phosphate in some lakes of Nainital, India. Tropical Ecology 22, 180-187.

Khulbe RD, Verma BL. 1983 - Aquatic fungi in some rice fields of tarai, Nainital. Tropical Plant Research 1(3), 268-270.

Kiran U, Sarkar N, Dayal R. 1982 - Pythium afertile a new record from India. Science and Culture. 48, 257-258.

Kirk PM, Cannon PF, Minter DW, Stalpers JA. 2008 - Ainsworth and Bisby’s Dictionary of the Fungi. 10th edn. Wallingford: CAB International.

Kumar A, Reeja ST, Suseela BR, Shiva KN. 2008 - Distribution of Pythium myriotylum Drechsler causing soft rot of ginger. Journal of Spices and Aromatic Crops 17(1), 5-10.

Kumar R, Gupta SK, Gupta M. 2018 - Investigation on etiology and epidemiology of damping-off of tomato seedlings in mid hills of Himachal Pradesh. Indian Phytopathology 71, 513-518.

Lévesque CA, de Cock AW. 2004 - Molecular phylogeny and taxonomy of the genus Pythium. Mycological Research 108, 1363-1383.

Lodha BC, Webster J. 1990 - Pythium acanthophoron, a mycoparasite rediscovered in India and Britain. Mycological Research 94, 1006.

Manoharachary C, Rao PH. 1978 - Distribution and seasonal variation of some Pythium sp. in some soils, pond water and mud soils of Hyderabad district. Mysore Journal of Agricultural Sciences 12, 280-285.

Manoharachary C, Reddy SM. 1975 - Syn-ecological studies on some Pythiaceous fungi from Andhra Pradesh. Indian Journal of Mycology and Plant Pathology 5, 199-101.

Martin FN, Loper JE. 1999 - Soil borne plant diseases caused by Pythium spp. ecology, epidemiology and prospects for biological control. Critical Reviews in Plant Sciences 18, 111-181.

Mathew R, Singh KK, Paul B. 2003 - Pythium campanulatum sp. nov., isolated from the rhizosphere of maize, its taxonomy, ITS region of rDNA, and comparison with related species. FEMS Microbiology Letters 226, 9-14. 
Mer GS. 1982 - Taxonomic and Physiological studies of Watermolds of Sat Tal (Nainital). Ph.D. Thesis, Kumaun University, Nainital.

Mer GS, Khulbe RD. 1983 - Pythium vexans var. minuta var. nov. from Kumaun, the Himalayas, India. Current Science 52 (15), 735-736.

Misra JK, Hall GS. 1996 - Occurrence and distribution of the genes Pythium in India, A review. Kavaka 24, 57-119.

Mufunda F, Muzhinji N, Sigobodhla T, Marunda M et al. 2017 - Characterization of Pythium spp. associated with root rot of tobacco seedlings produced using the float tray system in Zimbabwe. Journal of Phytopathology 165, 737-745.

Muthukumar A, Eswaran A, Nakkeeran S, Sangeetha G. 2010 - Efficacy of plant extracts and biocontrol agents against Pythium aphanidermatum inciting chilli damping-off. Crop Protection 29, 1483-1488.

Paul B, Bala K. 2008 - A new species of Pythium with inflated sporangia and coiled antheridia, isolated from India. FEMS Microbiology Letters 282(2), 251-257.

Prabhuji SK, Sinha SK. 1994 - Pythium multisporum Poitras: its Morphology and Phenology in the soils of Padrauna, India. Journal of Living World 1(2), 126-133.

Prabhuji SK, Srivastava GC. 1978 - Addition to lower fungi of India. Geobios 5, 35-36.

Raghunathan V. 1968 - Damping off of green gram, cauliflower, daincha, raghi and cluster bean. Indian Phytopathology 21, 456-457.

Rajagopalan S, Ramakrishnan K. 1964 - Phycomycetes in agricultural soils with special reference to Pythiaceae. I. Techniques of isolation. Journal of Madras University Section B 33, 311-341.

Rajagopalan S, Ramakrishnan K. 1971 - Phycomycetes in agricultural soils with special reference to the Pythiaceae. II. Species of Pythium isolated from Madras. Journal of Madras University Section B 37(38), 100-117.

Rajput KS, Koyani RD, Patel HR, Vasava AM et al. 2015 - A preliminary checklist of fungi of Gujarat State, India. Current Research in Environmental \& Applied Mycology 5(4), 285-306.

Rama Rao P. 1970 - Studies on soil fungi III. Seasonal variation and distribution of microfungi in some soils from Andhra Pradesh (India). Mycopathologia et Mycologia Applicata 40, 277298.

Ramakrishnan TS, Soumini CK. 1955 - Rhizome and root rot of turmeric caused by Pythium graminicola. Indian Phytopathology 7, 152-159.

Ramakrishnan K. 1955 - Some aspect of soil fungal ecology. Proceedings of the Indian Academy of Sciences 41B, 110-116.

Rao PR. 1970 - Studies on soil fungi IV. A comparison of some techniques for isolating soil fungi. Mycopathologia et Mycologia Applicata 40, 299-304.

Rao VG. 1963 - An account of the genus Pythium Pringsheim in India. Mycopathologia et Mycologia Applicata 21, 45-59.

Sarkar N, Chaurasia SHP, Dayal R. 1981 - Two new records of Pythium from India. National Academy of Science Letters 5, 187-188.

Sati SC. 1981 - Aquatic fungi of Kumaun in relation to fish infection. Ph.D. Thesis, Kumaun University, Nainital.

Sati SC. 1991 - Aquatic fungi parasitic on temperate fishes of Kumaun Himalaya, India. Mycoses 34, 437-441.

Sati SC, Tiwari N. 1992 - Studies on five species of Pythium parasitic on mustard and cabbage in India. Mycopathologia 119, 97-100.

Schurko AM, Mendoza L, de Cock AW, Bedard JE et al. 2004 - Development of a species-specific probe for Pythium insidiosum and the diagnosis of Pythiosis. Journal of Clinical Microbiology 42(6), 2411-2418.

Shanmugam V, Dohroo NP, Gupta M, Gangta V et al. 2010 - First report of Pythium splendens on ginger. International Journal of Plant Pathology 92, S109.

Sharma Y, Basu Chaudhary KC. 1981 - Rhizoctonia wilt, a new disease of cauliflower. Proceedings of National Academy of Sciences India 5IB, 204. 
Singh KK, Mathew R, Masih IE, Paul B. 2003 - ITS region of the rDNA of Pythium rhizosaccharum sp. nov. isolated from sugarcane roots: taxonomy and comparison with related species. FEMS Microbiology Letters 221(2), 233-236.

Singh SL, MS Pavgi. 1974 - Pythium middletonii parasitic in the roots of crucifers. Current Science 43, 351-352.

Srinivasan KV. 1956 - Pythium catenulatum Matthews causing sugar cane seedling root rot. Current Science 25, 299-300.

Srivastava DN, Rao VR. 1964 - Pythium stalk talk rot of corn in India. Current Science 33(4), 119120.

Srivastava GK, Ahmad A, Prabhuji SK. 2017 - Deep dermal tissue damage in Anabas testudineus Bl. parasitized by Pythium oligandrum Dreschler, Photon Journal of Pathology 105, 192-197.

Srivastava GK, Ahmad A, Prabhuji SK, Srivatava M et al. 2017 - Extracellular protease production by watermoulds determines their virulence during pathogenesis on fish. Journal of Advanced Zoology 38(1), 52-63.

Subramaniam LS. 1928 - Root rot and sclerotial diseases of wheat. Bulletin of Agricultural Research Institute Pusa 177, 1-17.

Sydow H, Butler EJ. 1907 - Fungi Indiae Orientalis. Annales Mycologici 483-515.

van der Plaats-Niterink AJ. 1981 - Monograph of the genus Pythium. Studies in Mycology 21, 1242.

van West P, Appiah AA, Gow NAR. 2003 - Advances in research on oomycete root pathogens. Physiological and Molecular Plant Pathology 62, 99-113.

Verma BL. 1984 - Studies on lower Phycomycetes in some agricultural fields of Tarai, Nainital with special reference to Saprolegniaceae and Pythiaceae. Ph.D. Thesis, Kumaun University, Nainital.

Verma BL. 1987a - Zoosporic fungi in wheat fields of Kumaun Himalaya. Madras Agricultural Journal 74(1), 1-5.

Verma BL. 1987b - A serious root disease of tomato caused by Pythium inflatum Matthews. Current Science 56, 616-617.

Verma BL, Khulbe RD. 1985 - Pythium polytylum Drechsler and Pythium rostratum Butler in some crop field of Tarai, Nainital. Science and Culture 51, 156-157.

Verma BL, Khulbe RD. 1986 - Pythium inflatum Mathews, a new record for India. Current Science $55,47-48$.

Villa NO, Kageyama K, Asano T, Suga H. 2006 - Phylogenetic relationships of Pythium and Phytophthora species based on ITS rDNA, cytochrome oxidase II and $\beta$-tubulin gene sequences. Mycologia 98, 410-422.

Webster J, Weber RWS. 2007 - Introduction to Fungi. 3rd edition. Cambridge University Press. Cambridge, UK.

Weiland JE, Beck BR, Davis A. 2012 - Pathogenicity and virulence of Pythium species obtained from forest nursery soils on Douglas-Fir seedlings. Plant Disease 97, 744-748.

Wilson KI, Rahim MA. 1978 - Pythium vexans causing fruit and rhizome rot of Aframomum melegueta. Indian Phytopathology 31, 238. 